\title{
VALORES DE DENSIDAD EN LA ESCALA DE GRISES DE MATERIALES RESTAURADORES MEDIANTE TOMOGRAFÍA COMPUTARIZADA DE HAZ CÓNICO. ESTUDIO PILOTO.
}

\section{Cone-beam computed tomography grayscale values for dental restorative materials. Pilot study.}

\begin{abstract}
Ericka María González Castro ${ }^{1}$, Guillermo Andrés Chaves Sojo $^{2}$, Deivi Antonio Cascante Sequeira ${ }^{3}$, Ana Cecilia Ruiz-Imbert*4.

${ }^{1}$ DCD, Práctica dental privada. Puntarenas, Costa Rica. ${ }^{2}$ Estudiante de la Licenciatura en Odontología, Facultad de Odontología, Universidad de Costa Rica, San José, Costa Rica. ${ }^{3}$ Estudiante de la Maestría en Radiología Oral, Escuela Dental de Piracicaba, Universidad de Campinas, São Paulo, Brasil. ${ }^{4}$ Docente del Departamento de Ciencias Diagnósticas y Quirúrgicas, Facultad de Odontología, Universidad de Costa Rica,

San José, Costa Rica. *anacecilia.ruiz@ucr.ac.cr
\end{abstract}

\section{Resumen}

Objetivos: Determinar el valor de densidad en la escala de grises (VDEG) de tres materiales de obturación coronaria, mediante Tomografía Computarizada de Haz Cónico (TCHC). Materiales y métodos: Se seleccionaron seis cabezas de cerdo y a cada una se le realizó una restauración coronaria en la pieza 7.2; utilizando uno de los siguientes materiales: amalgama, resina o Cention $\mathrm{N} 囚$, obteniendo dos muestras para cada material. Se adquirieron dos tomografías de cada espécimen, una de campo de visión pequeña $(4 \mathrm{x} 4 \mathrm{~cm})$ y otra de campo de visión grande $(8 \mathrm{x} 8 \mathrm{~cm})$, con parámetros de exposición fijos $(90 \mathrm{kV}, 5 \mathrm{mAs})$, para una muestra total de 12 tomografías. Se registraron los VDEG de los materiales para calcular el promedio de cada material. Resultados y conclusiones: Se observó que el VDEG promedio fue de 27 929,85 para amalgama; 5 798,54 para resina y 5885,47 para Cention $\mathrm{N} \circledast$. A pesar de que se observa una variación en los VDEG de cada material al cambiar el tamaño de campo, dicha diferencia no es estadísticamente significativa.

Palabras clave: Tomografía computarizada, Materiales dentales, Amalgama Dental, Resina Compuesta.

\begin{abstract}
Aim: To determine the grayscale value (GSV) of three coronary dental filling materials using Cone-Beam Computed Tomography (CBCT). Material and methods: Six pig heads were selected and a restoration in tooth 72 was performed using one of the following materials: amalgam, composite resin, or Cention $N \bowtie$, obtaining two samples of each material. Two CBCT acquisitions were acquired for each specimen, one with a small field of view $4 \times 4 \mathrm{~cm}$ ( and one with a large field of view $(8 x 8 \mathrm{~cm})$, with fixed exposure parameters $(90 \mathrm{kV}, 5 \mathrm{mAs})$, for a total sample of $12 \mathrm{CBCT}$ volumes. The GSV for each material was registered. Results and conclusions: It was observed that the average DGSV was 27929.85 for amalgam, 5798.54 for composite resin, and 5885.47 for Cention N®. Although there is a variation in the VDEG of each material when the field of view is modified, this difference was not statistically significant.
\end{abstract}

Key words: Cone-Beam Computed Tomography, Dental Materials, Dental Amalgam, Composite.

\section{Introducción}

La Tomografía Computarizada de Haz Cónico (TCHC) es una técnica de diagnóstico por imágenes que utiliza radiaciones ionizantes y produce imágenes tridimensionales del área maxilofacial o de otras regiones del cuerpo. ${ }^{1}$ En odontología, es de gran utilidad para el planeamiento de tratamiento y seguimiento en implantología, endodoncia, periodoncia, patología oral y cirugía maxilofacial. ${ }^{2}$

Las imágenes tomográficas pueden ser analizadas en un programa informático o software, dentro de los análisis cuantitativos que estos softwares permiten, se encuentra el 
grado de atenuación del haz de rayos $\mathrm{X}$ al interactuar con los tejidos. Este análisis genera valores numéricos conocidos como valores de la densidad en la escala de grises (VDEG) o números CT. ${ }^{1}$ Dichos valores se utilizaron inicialmente en las tomografías computarizadas de uso médico. En estos dispositivos se utiliza la escala de Unidades Hounsfield (UH) con rangos de valores estandarizados para tejidos de diferentes densidades, que permiten la identificación de los mismos de una manera objetiva. Sin embargo, para la TCHC los números CT no están estandarizados, por lo que no existe una escala que permita comparar los valores obtenidos. ${ }^{3-5}$

Con respecto a los materiales odontológicos, estos presentan diferentes densidades, dependiendo de su composición. De este modo, cuando estos materiales son expuestos a un haz de rayos $\mathrm{X}$, tienen diferentes capacidades de atenuación del mismo, lo que genera imágenes con variaciones en los tonos de grises que corresponden a distintos VDEG. ${ }^{6}$

El VDEG corresponde al rango de tonos en el vóxel, que es la unidad estructural de la tomografía. La cifra representa el coeficiente de atenuación de los rayos $\mathrm{X}$, y permite la evaluación de la densidad de los tejidos o materiales representados en la tomografía computarizada. Estos valores son diferentes, según el sistema tomográfico con el cual se hayan adquirido las imágenes. ${ }^{6}$

Debido a las diferencias según el modelo y el fabricante, existen parámetros de adquisición que pueden ser modificados en el equipo de acuerdo a la necesidad diagnóstica. Actualmente la mayoría de los equipos TCHC cuentan con parámetros energéticos ajustables, llámese potencial operativo $(\mathrm{kV})$ y la corriente del tubo-tiempo de exposición (mAs). Además los equipos de tomografía de haz cónico permiten variar el tamaño del volumen tomográfico o campo de visión (FOV, del inglés field of view), dependiendo de las necesidades clínicas o la tarea diagnóstica para la cual se indica dicho examen. ${ }^{7}$ De este modo lo tomógrafos pueden ofrecer imágenes circunscritas a la zona dentoalveolar (altura del campo de $10 \mathrm{~cm}$ o menos) o cubrir, inclusive, el área maxilofacial (altura superior a $10 \mathrm{~cm})^{8}$

Los tomógrafos de cobertura dentoalveolar, generalmente ofrecen campos de visión pequeños, donde se verán representados un sextante o una hemiarcada; y campos de visión grandes donde se incluye una arcada completa, ambos con la opción de abarcar uno de los rebordes (superior o inferior) o ambos (estudios bimaxilares). Las distintas dimensiones de altura y diámetro para los FOV varían entre las diferentes marcas y modelos de equipos. En general se recomienda utilizar el campo de visión más pequeño que se adapte a las necesidades clínicas, para reducir la dosis al paciente, disminuir la radiación dispersa y de este modo, mejorar la calidad de la imagen.,

Los VDEG resultan útiles al analizar tomografías, ya que podrían brindar una idea del grado de calcificación de una estructura y/o la naturaleza de un cuerpo extraño o material en los maxilares. Se desea evaluar los VDEG en el equipo Veraviewepocs ${ }^{\circledR}$ 3D R100 (J. Morita, Corp. Tokyo, Japón) para tres materiales de obturación coronaria (amalgama, resina compuesta, Cention $\mathrm{N} 囚$ ), en un modelo animal. Este estudio pretende servir como referencia para futuras etapas de investigación donde se culmine con la elaboración de una tabla referencial de VDEG específica para este equipo, como apoyo para el diagnóstico tomográfico.

\section{Materiales y métodos}

Este trabajo formó parte de un proyecto de investigación debidamente inscrito en la Vicerrectoría de Investigación Universitaria bajo el código B8368. Previo a la ejecución de este trabajo se consultó al Comité Institucional para el Cuidado y Uso de los Animales, quienes no tenían objeciones por tratarse de especímenes inanimados que se adquirieron en una carnicería.

\section{Preparación de la muestra:}

Se seleccionaron seis cabezas de cerdo,las cuales se cortaron de modo que pudieran ubicarse dentro del equipo de TCHC (diámetro menor a $17 \mathrm{~cm}$ ). Las mismas fueron adquiridas en una carnicería local y se almacenaron y transportaron en frío. En la cara vestibular de la pieza dental 72 de cada espécimen, se realizó una cavidad de 2 $\mathrm{mm}$ de profundidad y $2 \mathrm{~mm}$ de diámetro, utilizando una pieza de mano de alta velocidad y una broca de carburo. Posteriormente se obturó cada cavidad con un material de obturación diferente (resina, amalgama y Cention $\mathrm{N} \circledast$ ). Se distribuyó la muestra de manera que se tuvieran dos cavidades obturadas con cada material.

\section{Procedimiento de preparación para cada material:}

Resina: Obturación con resina Filtek P60 3M®: Grabado con ácido fosfórico al $35 \%$, limpieza de la cavidad con Clorhexidina al $2 \%$, colocación de dos capas de adhesivo Single Bond $3 \mathrm{M} \circledast$ de acuerdo a las indicaciones del fabricante, se fotocuró un único incremento usando una lámpara de fotocurado marca Gnatus ${ }^{\circledR}$, durante 40 segundos.

- Amalgama: Se utilizó la amalgama de la marca $\mathrm{Nu}$ Alloy®, se realizó una preparación retentiva, se limpió la cavidad con clorhexidina al $2 \%$. Se colocó la cápsula en el amalgamador por 10 segundos, se empacó con 
el instrumento en la cavidad preparada y se bruñó, retirando los excesos.

- Cention N®: Se dispensó líquido y polvo en loseta de papel, se mezcló hasta obtener una consistencia homogénea y manipulable; con ayuda de un instrumento se transportó a la cavidad, se removieron los excesos y se esperó 4 minutos para la polimerización.

\section{Adquisición de las imágenes:}

Usando el equipo tomográfico Veraviewepocs® 3D R100, se adquirieron dos tomografías de cada espécimen, con los parámetros de exposición de $90 \mathrm{kV}, 5 \mathrm{~mA}$, un tiempo de adquisición de $9.4 \mathrm{~s}$.

Se realizó una vista de prueba o "scout", para adecuar el campo de visión a la zona de interés. Luego, se adquirió cada volumen tomográfico, uno de campo de visión grande $(8 \times 8 \mathrm{~cm})$ y otro de campo de visión pequeño $(4 \times 4 \mathrm{~cm})$, obteniendo una muestra de doce tomografías en total. Cada espécimen fue posicionado de manera que el diente de interés estuviese en la posición más centrada posible dentro del campo de visión.

\section{Evaluación de las imágenes:}

Se realizó la calibración de cinco evaluadores con una especialista en radiología oral y maxilofacial, obteniéndose un coeficiente de correlación intraclase (ICC) interevaluadores alto (0.952). Durante esta calibración se los capacitó en el manejo del programa informático propio del equipo, i-Dixel, para la evaluación de las tomografías. Durante la calibración se realizó la revisión de cinco tomografías y el registro de los VDEG en puntos específicos de cada una de ellas y fueron comparadas con los parámetros tomados por la especialista.

Se elaboró un instrumento de recolección de datos para registrar los VDEG de los materiales. Cinco operadores evaluaron las tomografías en la sala de interpretación de la sección de radiología oral de una clínica dental universitaria, en un ambiente silencioso y oscuro, utilizando un monitor con las características HP Pro Desk 600 G3 SFF. Acorde a las recomendaciones de la Guía para el Uso Seguro del TCHC, ${ }^{9}$ las evaluaciones se realizaron durante las tardes, en un horario comprendido entre las 13:00 y 16:00 horas para evitar fatiga visual, ${ }^{10}$ durante un período máximo de 30-45 minutos.

Utilizando el software, se registraron los VDEG de cada material en ambas tomografías (campo grande y campo pequeño), en la ventana sagital maximizada a 400X, seleccionando con el cursor un área menor o igual a $0,5 \mathrm{~mm}^{2}$; que incluyera solamente el material obturador a evaluar. (Figura 1)

\section{Análisis estadístico:}

Se realizó el ICC para observar la concordancia intervaluadores. Mediante estadística descriptiva se calculó el valor promedio, mediana, mínimo, máximo y desviación estándar de los valores de densidad en la escala de grises de los materiales mencionados anteriormente. 


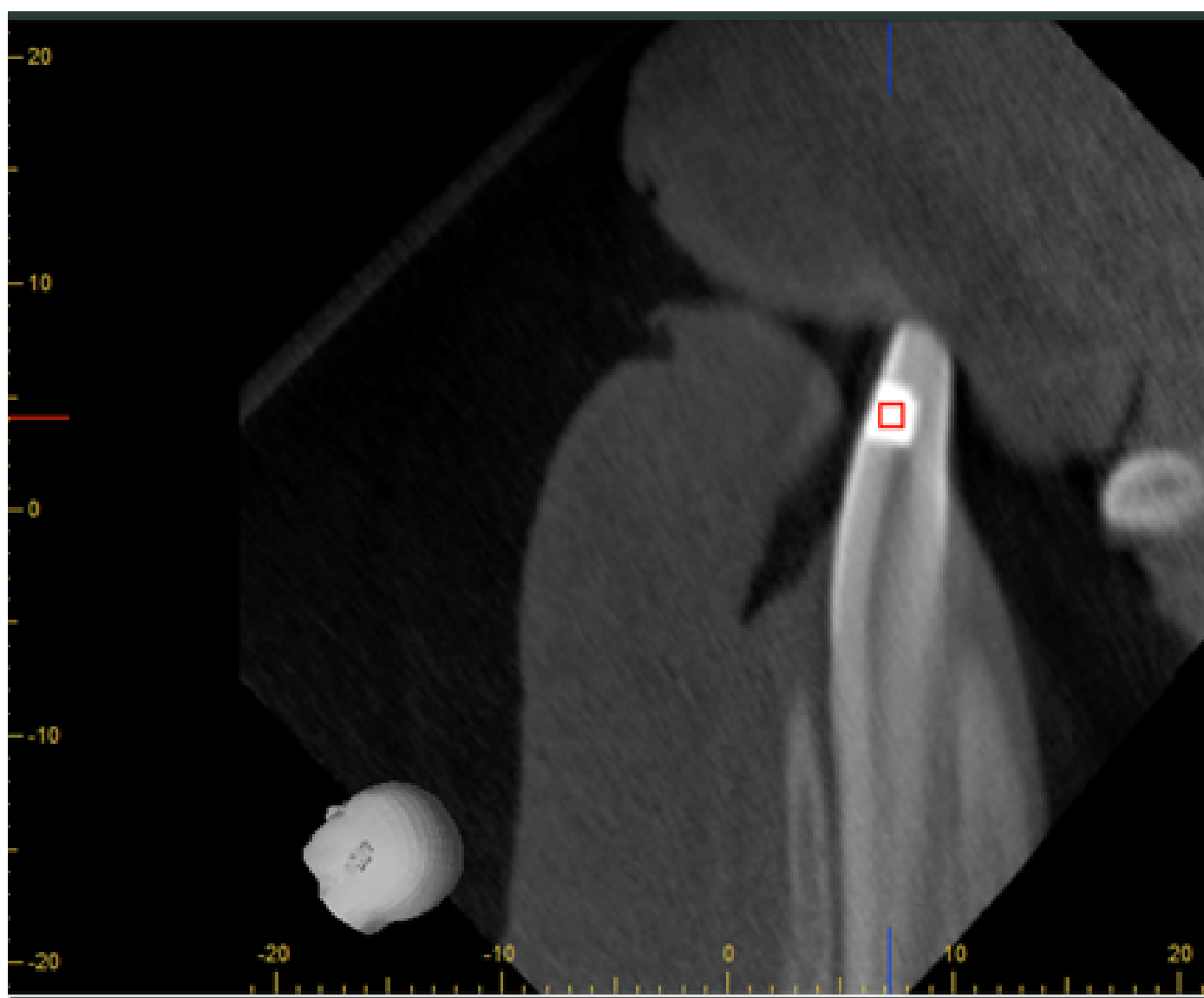

$n$ Grayscale $n$ Color Presets Image Info/Profiles

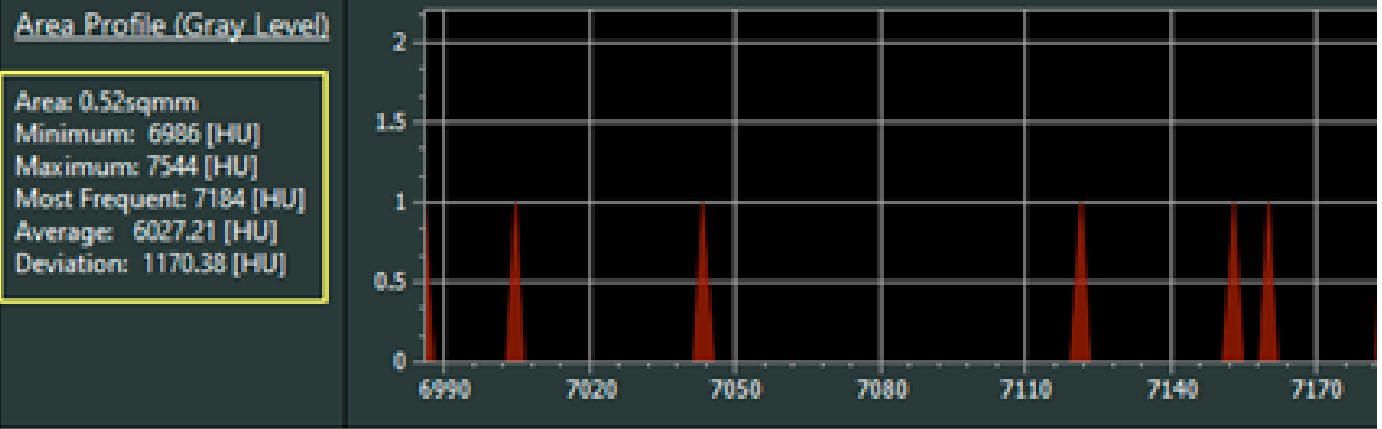

Fig. 1. Determinación de la Región de Interés (ROI) en un corte sagital para la medición del valor de densidad en la escala de grises usando el software i-Dixel.

\section{Resultados}

Los evaluadores realizaron 12 mediciones de los VDEG de las tomografías, para un total de 60 registros. Los operadores fueron calibrados previamente con un nivel de concordancia del 0,952 .

Con la evaluación de la escala de grises, según el material, se comprobó que la amalgama tuvo un comportamiento diferente a la resina y al Cention $\mathrm{N} \circledast$ $(\mathrm{p}=0,001)$ con la prueba de Jonckheere-Terpstra; debido a que no se cumplieron los supuestos de homogeneidad de varianza ni el supuesto de normalidad para utilizar el análisis de varianza.

En la Tabla 1 se muestran los valores obtenidos de promedio, mediana, mínimo, máximo y desviación estándar, para cada material.

En la tabla 2 se observan los valores VDEG del mismo 


\begin{tabular}{lrrr}
\hline & \multicolumn{3}{c}{ Material } \\
Estadísticas & Amalgama & Resina & Cention N \\
\hline Media & $27.929,85$ & $5.798,54$ & $5.885,47$ \\
Mediana & $27.308,73$ & $5.505,34$ & $6.171,64$ \\
Desviación estándar & $9.503,18$ & $1.149,16$ & $1.480,03$ \\
Mínimo & $13.640,37$ & $3.618,38$ & $2.808,15$ \\
Máximo & $48.838,88$ & $8.254,78$ & $8.266,27$ \\
\hline
\end{tabular}

Tabla 1. Valores estadísticos obtenidos de las mediciones de densidad en la escala de grises para los diferentes materiales utilizados.

material según el tamaño de la tomografía, sea esta pequeña $(4 \times 4 \mathrm{~cm})$ o grande $(8 \times 8 \mathrm{~cm})$.

\begin{tabular}{ccc}
\hline & \multicolumn{2}{c}{ Campo } \\
& Pequeño & Grande \\
& & \\
\hline Media & $15.373,50$ & $11.035,74$ \\
Mediana & $6.977,97$ & $6.280,28$ \\
Desviación estándar & $14.257,64$ & $8.530,45$ \\
Mínimo & $2.808,15$ & $3.321,69$ \\
\hline Máximo & $48.838,88$ & $29.422,09$ \\
\hline
\end{tabular}

Tabla 2. Valores de densidad en la escala de grises de los materiales según el tamaño de campo.

Se puede observar que los valores obtenidos de densidad en la escala de grises fueron distintos para el mismo material, dependiendo del tamaño del campo de visión de la tomografía (pequeño o grande). Sin embargo, esta diferencia no resultó significativa $(\mathrm{p}=0,261)$ de acuerdo con la prueba U de Mann-Whitney. No se utilizó el análisis de varianza debido a que no se cumplieron los supuestos de homogeneidad de varianza ni tampoco el supuesto de normalidad.

\section{Discusión}

Varios autores han utilizado cabezas de animales para el estudio de los VDEG en TCHC, ya sean $\operatorname{cerdos}^{11,12} \mathrm{u}$ ovejas. La colocación de los materiales en piezas dentales de los especímenes, permite asemejarse más al medio oral, ya que se conserva el hueso alveolar, la gíngiva, los músculos y demás tejidos normalmente presentes en condiciones clínicas. Es importante recordar que el haz de rayos $\mathrm{X}$ interactúa con todos estos tejidos y produce radiación dispersa, la cual alcanza el receptor de imagen y tiene influencia en la imagen final. ${ }^{6}$ De este modo se puede realizar investigación con radiaciones ionizantes controlando las variables, sin exposición innecesaria a seres humanos.

Con respecto a la elección de los materiales restauradores a evaluar, se seleccionó la amalgama y la resina compuesta al ser de uso frecuente. Sin embargo, se incluyó el Cention $\mathrm{N} \circledast$, como una opción emergente sobre la que hay poca investigación en TCHC. Este es un material resinoso de reciente introducción en el mercado; es de relleno alcalino a base de alcasites capaz de liberar iones hidróxido en la superficie dentaria, se coloca en incrementos en cavidades retentivas; es estético, resistente a la flexión y de curado dual. ${ }^{13-15}$

Se estima que el uso de la amalgama puede disminuir en Costa Rica, debido a que el país se suscribió al Convenio de Minamata, un tratado global que pretende controlar y mitigar las emisiones y liberaciones de mercurio y sus compuestos. Esto implica que el país se comprometió a disminuir las emisiones de este elemento, que es parte de la aleación de la amalgama dental. ${ }^{16}$ Sin embargo, debido a la alta durabilidad de las restauraciones de amalgama, ${ }^{17}$ este material podrá encontrarse en las tomografías dentales de los pacientes en el largo plazo, por lo que el estudio de sus características tomográficas aún es relevante.

Con respecto a los valores de densidad en la escala de grises obtenidos en las tomografías de los materiales evaluados, se obtuvo para la amalgama un valor notablemente superior a la resina y al Cention $\mathrm{N} 囚$. Varios autores concuerdan en que la amalgama dental fue el material que presentó un mayor VDEG; ${ }^{18,19}$ con un valor promedio de 15 $383,0^{18}$ y 11253,$70 ;{ }^{19}$ en comparación con otros materiales como la resina compuesta, la cual obtuvo un 6896,0 (18) y $8217,50^{19}$ de media. Estos valores discrepan a los obtenidos en este estudio debido a que fueron captados con equipos diferentes.

Para Hadadi y colaboradores, ${ }^{19}$ la amalgama fue el único material que mostró diferencia estadísticamente significativa en el VDEG con respecto a los otros materiales (resina compuesta, resina fluida, ionómero de vidrio y Dycal), lo cual concuerda con los hallazgos de este estudio. 
Para la resina compuesta, los VDEG obtenidos $(5$ 798,54) discrepan de los hallazgos de Rizo, ${ }^{20}$ quien evaluó la misma marca de material en el mismo equipo y obtuvo un valor de 1 646,9. Esta discrepancia se puede relacionar al uso de diferentes parámetros de adquisición $(80 \mathrm{kVp}-5 \mathrm{~mA})$ y al hecho de que su estudio se realizó en un simulador de PMMA, y no en un medio oral animal.

El comportamiento de los VDEG de los diferentes materiales es esperable en relación con la naturaleza de sus componentes. Los materiales son más radiopacos o hiperdensos a mayor número atómico y grosor. $^{6} \mathrm{La}$ amalgama es una aleación metálica cuyos componentes principales poseen los siguientes números atómicos: $47=$ Plata, 50=Estaño, $29=$ Cobre, $30=Z$ Zinc y $80=$ Mercurio.${ }^{17}$ Se deduce que la amalgama va a tener mayor VDEG que la resina o el Cention $\mathrm{N} 囚$, porque los números atómicos de los componentes radiopacos de estos dos materiales son más bajos que los de la amalgama $(B a r i o=56$, Estroncio $=38$, Zirconio $=40) ;{ }^{21}$ es decir, que estos son menos densos $\mathrm{y}$, por ende, con menor valor en la escala de grises.

Respecto al efecto del tamaño de campo en el VDEG, se observaron diferencias para los mismos materiales según estuvieran en un campo grande o pequeño. De manera general, se observaron valores de DEG más altos para los campos pequeños y más bajos para campos grandes. Sin embargo, dichas diferencias no fueron estadísticamente significativas $(p=0,261)$, por lo que es necesario realizar más estudios con un tamaño muestral mayor, para probar esta relación. Shokri y colaboradores ${ }^{22}$ estudiaron el efecto que tiene el utilizar dos tamaños de campos diferentes en el valor de escala de grises. Hicieron la adquisición volumétrica en dos diferentes equipos tomográficos de haz cónico, usando dos tamaños de campo. Los resultados mostraron una diferencia significativa $(\mathrm{p}=0,05)$ en los valores promedio de escala de grises según el tamaño de campo.

Parsa y colaboradores $^{23}$ buscaron demostrar la influencia de diferentes parámetros de escaneo de la TCHC en los VDEG, siendo uno de dichos parámetros el tamaño de campo de visión. Utilizaron dos equipos distintos, Accuitomo y NewTom, el primero mostró un aumento en el VDEG con el aumento del tamaño de campo, mientras que el segundo obtuvo una disminución constante en los VDEG cuando el tamaño de campo aumentó. Este segundo comportamiento se asemeja a los resultados de este estudio, donde tamaños de campo grandes mostraron VDEG menores para el mismo material.

El tamaño del campo de visión es un factor de escaneo determinante para la calidad de la imagen tomográfica. ${ }^{23}$ El ruido, es un artificio tomográfico de origen físico que disminuye la resolución de bajo contraste en las imágenes de TCHC. Una de las fuentes de ruido en TCHC es la radiación dispersa, la cual se origina cuando los fotones del haz de rayos $\mathrm{X}$ interactúan con los átomos del objeto a radiografiar y son desviados de su trayectoria original. ${ }^{24}$ Una vez que los fotones desviados alcanzan el receptor de imagen, son interpretados erróneamente por los algoritmos durante la reconstrucción ya que asumen una trayectoria lineal. Esta radiación dispersa depende del tamaño, la forma y la posición en el campo de visión del objeto, así como de los parámetros energéticos. ${ }^{24}$ Un campo de visión más pequeño disminuye la cantidad de radiación dispersa, por tanto tiene impacto positivo en la calidad de imagen, lo que se puede observar subjetivamente como menor ruido. ${ }^{8}$ Esto podría explicar la diferencia en los VDEG entre campos de visión grandes y pequeños, tal como se encontró en este estudio. En contraste, otros autores afirman que las tomografías con campos de visión más grandes presentan menor cantidad de ruido, probablemente debido a una reducción en la exomasa, es decir, en la cantidad de estructuras ubicadas fuera del FOV pero entre el origen del haz de rayos X y el receptor de imagen. ${ }^{25}$ Inclusive, objetos de alta densidad ubicados en la exomasa, es decir, fuera del campo de visión de la tomografía, tienen efecto en los VDEG, siendo mayor su efecto en el centro del FOV, donde los VDEG disminuyen. ${ }^{25}$ En el caso de la muestra utilizada en este estudio, no se descarta la influencia de las estructuras en la exomasa en los valores registrados de VDEG; ya que se evaluaron cabezas animales seccionadas, que incluían tejidos duros y blandos más allá del área dentoalveolar.

Los hallazgos encontrados en el presente estudio piloto y la evidencia de la literatura, mostraron cómo los VDEG son sensibles a varios parámetros técnicos de la toma, por lo que se debe realizar investigación en este campo, que sea específica para cada equipo y considerando los diferentes parámetros técnicos, antes de darle uso clínico a estas cifras.

Se recomienda usar los datos obtenidos para calcular un tamaño muestral mayor, que permita estudiar la relación entre el tamaño de campo y los valores de densidad en la escala de grises para cada material.

Debido a la gran diversidad que existe en los equipos para tomografía computarizada de haz cónico, es importante definir los valores de densidad en la escala de grises específicos para cada uno; teniendo en cuenta factores técnicos como el kilovoltaje, miliamperaje, resolución y tamaño de campo y no comparar resultados entre equipos o con estudios realizados utilizando distintos parámetros. Asimismo, los autores sugieren el uso de cabezas de otro animal de granja de menores dimensiones, como las cabras u ovejas, ya que se enfrentó cierta dificultad para realizar el corte de las cabezas de cerdo a las dimensiones necesarias para ser posicionadas en el equipo a la hora de las adquisiciones tomográficas. 


\section{Conclusiones}

El valor promedio de la densidad en la escala de grises de la amalgama dental fue de 27 929,85. Esta cifra fue la mayor de todos los materiales y la diferencia en su valor fue estadísticamente significativa respecto a los demás.

El valor promedio de la densidad en la escala de grises de la resina compuesta fue de 5798,54 ; y para el Cention $\mathrm{N} \circledast$ fue de 5885,47 . A pesar de que se observó una variación en los valores de escala de grises de cada material al cambiar el tamaño de campo, dicha diferencia no fue estadísticamente significativa.

Conflicto de intereses: Los autores del presente estudio manifiestan que no existe ningún conflicto de intereses en relación al tema de estudio.

Agradecimientos : A la Facultad de Odontología de la Universidad de Costa Rica, quienes facilitaron las instalaciones, los equipos y los materiales para realizar la investigación, así como al personal técnico asistencial por su apoyo durante la preparación y adquisición de las muestras. A la MSc. Jaqueline Castillo, por su asesoramiento estadístico.

\section{Referencias}

1 Sarment D. Cone Beam Computed Tomography: Oral and Maxillofacial Diagnosis and Applications. Estados Unidos. John Wiley \& Sons, Inc; 2014.

2 Manoj S, Chandra P E, Kailasam S, Raghuram PH, Sateesh S, Karpagavalli. Applications of Cone-Beam Computed Tomography in dentistry. J. Indian Acad. Oral Med. Radiol. 2011; 23(4): 163-167.

3 Miles D, Danforth R. A Clinician's Guide to Understanding Cone Beam Volumetric Imaging (CBVI). The Academy of Dental Therapeutics and Stomatology. 2008; 1-15.

4 Molteni R. Prospects and challenges of rendering tissue density in Hounsfield units for cone beam computed tomography. Oral and Maxillofacial Radiology. 2013; 116 (1): 105-119.

5 Oliveira M, Tosoni G, Lindsey D et al. Assessment of CT numbers in limited and medium field-of-view scans taken using accuitomo 170 and veraviewepocs 3De conebeam computed tomography scanners. Imaging Science in Dentistry, Imaging Science in Dentistry. 2014; 44: 279-285.

6 White S, Pharoah M. Radiología Oral, Principios e Interpretación. 4ta Edición. España Editorial Harcourt, S.A; 2002.

7 Ruiz-Imbert A ,Cascante-Sequeira D. Valores de densidad en la escala de grises en Tomografía Computarizada de Haz Cónico: Alcances y limitaciones. International Journal of Dental Sciences. 2020; DOI 10.15517/IJDS.2021.45106.

8 EUROPEAN COMMISSION. Radiation Protection $\mathrm{N}^{\circ}$ 172: Cone beam CT for dental and maxillofacial radiology. Evidence-based guidelines.
2012 [citado 10 de diciembre del 2020]. http://www.sedentexct.eu/files/radiation_protection_172.pdf 9 Centre for Radiation Agency. Guidance on the Safe Use of Dental Cone Beam CT (Computed Tomography) Equipment. HPA-CRCE, 2010; 10(1): 1-64.

10 Stec N, Arje D, Moody E, Krupinski N, Pascal N. A Systematic Review of Fatigue in Radiology: Is It a Problem? American Journal of Roentgenology, 2018; 210(4): 799806.

11 Lopes P, Santaella G, Lima C, Vasconcelos K, Groppo F. Evaluación of soft-tissues simulant materials in cone beam computed tomography. Dentomaxillofacial Radiology. 2018; 48 (1) :1-8.

12 Razi T, Niknami M, Alavi Ghazani F. Relationship between Hounsfield Unit in CT Scan and Gray Scale in CBCT. J Dent Res Dent Clin Dent Prospects [Internet]. 2014; 8 (2): 10710.

13 Kaur M, Singh N, Jhamb A, Batra D, A comparative evaluation of compressive strength of Cention $\mathrm{N}$ with glass Ionomer cement: An in-vitro study, International Journal of Applied Dental Sciences 2019; 5(1): 5-9.

14 Deepak S, Nivedhitha MS, Proximal contact tightness between two different restorative materials - An in vitro study. Journal of Advanced Pharmacy Education \& Research, 2017; 7(2): 153-156.

15 Singh J, Sharma S, Maurya S, Suman A. CENTION N: A REVIEW . International journal of current research, 2018, 10(5): 69111-69112.

16 Fernández A, Herrera J, Sibaja J. Perfil nacional de uso de mercurio en Costa Rica a la luz de la entrada en vigencia del Convenio de Minamata. Revista de Ciencias Ambientales. 2017; 51(2): 145-168.

17 Bharti R, Kaur K, Prakash A, Chandra A. Dental Amalgam: An Update. Journal of Conservative Dentistry. 2010; 13(4): 204-208.

18 Emadi N, Safi Y, Akbarzadeh A, Asgary S. Comparison of CT-Number and Gray Scale Value of Different Dental Materials and Hard Tissues in CT and CBCT. Iranian Endodontic Journal. 2014; 9(4): 283-286.

19 Hadadi P, Ostovarrad F, Nikbin A, Ranjzad H, Ghasemi F, Nemati S. Evaluation of the gray level of restorative materials using cone-beam computed tomography: A crosssectional study. Dental and Medical Problems. 2018; 55(3): 267-274.

20 Rizo S. Desarrollo y construcción de un maniquí de calidad de imagen para tomógrafos dentales CBCT (Tesis de maestría). Costa Rica: Universidad de Costa Rica. 2018.

21 Arango J, Gómez J, Muñoz I, Rojas E, Sinisterra G, Moreno $\mathrm{S}$, Moreno F. Evaluación radiográfica de dientes con restauraciones clase I en resina compuesta sometidos a altas temperaturas. Journal Odontológico Colegial. 2020; 13(25):8-25.

22 Shokri A, Ramenazi L, Bidgoli M, Akbarzadeh M, Ghazikhanly-Sani K, Fallahi-Sichani H. Effect of field-of- 
view size on gray values derived from cone-beam computed tomography compared with the Hounsfield unit values from multidetector computed tomography scans Imaging. Science in Dentistry. 2018; 48(1): 9-31.

23 Parsa A, Ibrahim N, Hassan B, Motroni A, van der Stelt $\mathrm{P}$, Wismeijer D. Influence of cone beam CT scanning parameters on grey value measurements at an implant site. Dentomaxillofac Radiol. 2013; 42(1): 1-7.

24 Jaju P, Jain M, Singh A, Gupta A. Artefacts in cone beam CT. Open Journal of Stomatology. 2013; 3: 292-297.

25 Candemil A, Salmón B, Queiroz Freitas D, Haiter Neto F, Lima Oliveira M. Distribution of metal artifacts arising from the exomass in small field-of-view cone beam computed tomography scans. Oral Surg Oral Med Oral Pathol Oral Radiol 2020;130:116-125.

Recibido: 19 de diciembre de 2020

Aceptado: 22 de febrero de 2021 